\title{
GROUP ALGEBRA MODULES. IV
}

\author{
BY \\ S. L. GULiCK( $\left.{ }^{(}\right)$, T.-S. LIU( $\left.{ }^{1}\right)$ AND A. C. M. van ROOIJ
}

\begin{abstract}
Let $\Gamma$ be a locally compact group, $\Omega$ a measurable subset of $\Gamma$, and let $L_{\Omega}$ denote the subspace of $L^{1}(\Gamma)$ consisting of all functions vanishing off $\Omega$. Assume that $L_{\Omega}$ is a subalgebra of $L^{1}(\Gamma)$. We discuss the collection $\Re_{\Omega}(K)$ of all module homomorphisms from $L_{\Omega}$ into an arbitrary Banach space $K$ which is simultaneously a left $L^{1}(\Gamma)$ module. We prove that $\Re_{\Omega}(K)=\Re_{\Omega}\left(K_{0}\right) \oplus \Re_{\Omega}\left(K_{\mathrm{abs}}\right)$, where $K_{0}$ is the collection of all $k \in K$ such that $f k=0$, for all $f \in L^{1}(\Gamma)$, and where $K_{\text {abs }}$ consists of all elements of $K$ which can be factored with respect to the module composition. We prove that $\Re_{\Omega}\left(K_{0}\right)$ is the collection of linear continuous maps from $L_{\Omega}$ to $K_{0}$ which are zero on a certain measurable subset of $X$. We reduce the determination of $\Re_{\Omega}\left(K_{\mathrm{abs}}\right)$ to the determination of $\Re_{\Gamma}\left(K_{\mathrm{abs}}\right)$. Denoting the topological conjugate space of $K$ by $K^{*}$, we prove that $\left(K_{\mathrm{abs}}\right)^{*}$ is isometrically isomorphic to $\Re_{\Omega}\left(K^{*}\right)$. Finally, we discuss module homomorphisms $R$ from $L_{\Omega}$ into $L^{1}(X)$ such that for each $f \in L_{\Omega}, R f$ vanishes off $Y$.
\end{abstract}

1. Introduction. Once again we come back to the question of module homomorphisms which began our investigation of group algebra modules in the first place ([3] and [4]). The present paper descends from both these papers. If $\Gamma$ is a locally compact group, $L^{1}(\Gamma)$ the Banach space of integrable functions on $\Gamma$, and if $K$ is a left $L^{1}(\Gamma)$ module, we studied in [3] the collection of module homomorphisms from $L^{1}(\Gamma)$ into $K$, from a rather abstract vantage point. On the other hand, if $\Gamma$ acts on a locally compact space $X$ as a transformation group, $m_{X}$ is a positive Radon measure on $X$ quasi-invariant with respect to $\Gamma$, and if $L^{1}(X)$ is the Banach space of integrable functions on $X$, we showed in [4] that $L^{1}(X)$ can be made into a left $L^{1}(\Gamma)$ module, and then we examined the module homomorphisms from $L^{1}(\Gamma)$ into $L^{1}(X)$.

In the present paper we let $\Omega$ be a measurable subset of $\Gamma$, and let $L_{\Omega}$ denote the subspace of $L^{1}(\Gamma)$ consisting of all functions vanishing off $\Omega$. We assume that $L_{\Omega}$ is a subalgebra of $L^{1}(\Gamma)$. Then we discuss the module homomorphisms from $L_{\Omega}$ into an arbitrary left $L^{1}(\Gamma)$ module $K$. The collection of such homomorphisms we call $\Re_{\Omega}(K)$. The fact that $L_{\Omega}$ need not have an approximate identity makes the

Received by the editors June 17, 1969.

AMS 1969 subject classifications. Primary 4680, 2220.

Key words and phrases. Transformation group, quasi-invariant measure, absolutely continuous measure, approximate identity, factorable, group algebra module, module homomorphism, absolutely continuous module, order-free module, subsemigroup, left-translation invariant, orbit topology.

${ }^{(1)}$ Research supported in part by the National Science Foundation.

Copyright (C) 1970, American Mathematical Society 
problem much more difficult. The fact that $L_{\Omega}$ has shifted approximate identities makes the problem solvable, via several reductions.

In $\S 3$ we prove that $\Re_{\Omega}(K)=\Re_{\Omega}\left(K_{0}\right)+\Re_{\Omega}\left(K_{\text {abs }}\right)$, where $K_{0}$ consists of all elements of $K$ which when composed with elements of $L^{1}(\Gamma)$ yield the zero element in $K$, and where $K_{\text {abs }}$ consists of all elements of $K$ which can be factored into the composition of an $L^{1}(\Gamma)$ element and some element of $K$. Thus the homomorphism problem splits into two parts. Via Theorem $3.5, \Re_{\Omega}\left(K_{0}\right)$ is the collection of linear, continuous maps from $L_{\Omega}$ to $K_{0}$ which are zero on $L_{T}$ where $T$ is a certain measurable subset of $G$; this set $T$ also turns up in the previous paper [5], it involves the composition operator in a direct way.

Next in $\S 4$, we look at $\Re_{\Omega}\left(K_{\text {abs }}\right)$. Let $d \Omega=\{\sigma \in \Gamma$ : for every measurable neighborhood $\Phi$ of $\sigma, \Phi \cap \Omega$ has positive measure $\}$. We show that $d \Omega$ splits up into a collection $\mathscr{J}(d \Omega)$ of pairwise disjoint subsets. Then each $R \in \Re_{\Omega}\left(K_{\mathrm{abs}}\right)$ corresponds to a collection $\left(R_{J}\right)_{J \in \mathscr{I}(d \Omega)}$ where $R_{J} \in \Re_{\Gamma}\left(K_{\text {abs }}\right)$ and such that $R_{J}$ and $R$ are identical on $\Omega \cap J$. Furthermore, for each $J$, the homomorphism $R_{J}$ is uniquely defined. Conversely, any collection $\left(R_{J}\right)_{J \in \mathscr{I}(d \Omega)}$ which is norm-bounded in $\Re_{\Gamma}(K)$ gives rise to a (unique) $R \in \Re_{\Omega}(K)$. Thus the problem of finding $\Re_{\Omega}\left(K_{\mathrm{abs}}\right)$ has reduced to that of finding $\Re_{\Gamma}\left(K_{\text {abs }}\right)$.

In $\S 5$ we assume that $\Omega=\Gamma$. We embed $K$ into $\Re_{\Gamma}(K)$ by the map $T_{K}$ which sends $k \in K$ into right module multiplication by $k$. Then $K_{\text {abs }}$ is injected isometrically onto $\left[\Re_{\Gamma}(K)\right]_{\text {abs. }}$. Denoting the topological conjugate space of $K$ by $K^{*}$, we prove that $\left(K_{\text {abs }}\right)^{*}$ is isometrically isomorphic as a module to $\Re_{\Gamma}\left(K^{*}\right)$. This paves the way for a collection of examples.

Let $Y \subseteq X$ be measurable and let $\mathscr{J}(d \Omega)$ consist of only one element. We close the paper with a study of the module homomorphisms $R$ from $L_{\Omega}$ to $L^{1}(X)$ such that for each $f \in L_{\Omega}, R f$ vanishes off $Y$.

2. Notations. The notations we use are mainly those given in $\$ 2$ of [5]. Most of the definitions and comments below have already appeared in our preceding texts, but we desire to have them stated here explicitly for reference.

Let $\Gamma$ be a locally compact group with identity 1 and left Haar measure $m$. For $f \in L^{1}(\Gamma)$ and $\sigma \in \Gamma$ we have $L^{1}$-functions $f_{\sigma}, f^{\sigma}, f^{\prime}$ defined by

$$
\begin{array}{lll}
f_{\sigma}(\tau)=f(\sigma \tau), & & \tau \in \Gamma, \\
f^{\sigma}(\tau)=f(\tau \sigma) \Delta(\sigma), & & \tau \in \Gamma, \\
f^{\prime}(\tau)=f\left(\tau^{-1}\right) \Delta\left(\tau^{-1}\right), & & \tau \in \Gamma .
\end{array}
$$

These functions are connected with the convolution in $L^{1}(\Gamma)$ by the formulas $f_{\sigma} * g=(f * g)_{\sigma}, f^{\sigma} * g=f * g_{\sigma}, f * g^{\sigma}=(f * g)^{\sigma}$, and $(f * g)^{\prime}=g^{\prime} * f^{\prime}\left(f, g \in L^{1}(\Gamma)\right)$.

Let $\Omega \subseteq \Gamma$ be measurable. We put $L_{\Omega}=\left\{f \in L^{1}(\Gamma): f=0\right.$ a.e. outside $\left.\Omega\right\}$ and $d \Omega=\{\sigma \in \Gamma:$ for every measurable neighborhood $\Phi$ of $\sigma, m(\Phi \cap \Omega) \neq 0\}$. Then $\Omega \subseteq d \Omega$ 1.a.e. (= locally almost everywhere), i.e. $d \Omega \mid \Omega$ is locally null. For every $\sigma \in d \Omega, L_{\Gamma}$ contains approximate identities $\left(u_{i}\right)_{i \in I}$ and $\left(v_{j}\right)_{j \in J}$ such that $\left(u_{i}\right)^{\sigma^{-1} \in L_{\Omega}}$ for each $i$ and $\left(v_{j}\right)_{\sigma^{-1}} \in L_{\Omega}$ for each $j$ [5, Lemma 3.1]. 
For measurable $\Omega, \Phi \subseteq \Gamma$, we let $L_{\Omega} * L_{\Phi}$ be the closed linear hull of $\left\{f * g: f \in L_{\Omega}, g \in L_{\oplus}\right\}$. Then

$$
L_{\Omega} * L_{\Phi}=\mathrm{Cl}\left(\sum_{\sigma \in d \Omega} L_{\sigma \Phi}\right)=\mathrm{Cl}\left(\sum_{\tau \in d \Phi} L_{\Omega \tau}\right) .
$$

(The first equation is [5, Corollary 3.4]; the second is proved in a similar way as the first.) If $\Phi$ is not locally null, then $d \Phi \neq \varnothing$; taking $\Omega=\Gamma$ we obtain from (2.1) that

$$
\mathrm{Cl}\left(\sum_{\sigma \in \Gamma} L_{\sigma \oplus}\right)=L_{\Gamma}
$$

In case $L_{\Omega}$ is a subalgebra of $L_{\Gamma}$ we obtain from (2.1) the inclusions $\Omega \sigma \subseteq \Omega$ 1.a.e. and $\sigma \Omega \subseteq \Omega$ 1.a.e. for all $\sigma \in d \Omega$. Thus, if $f \in L_{\Omega}$ and $\sigma \in d \Omega$, then $f^{\sigma^{-1}} \in L_{\Omega}$ and $f_{\sigma^{-1}} \in L_{\Omega}$. By [5, Corollary 3.5(ii)] $d \Omega$ is a closed semigroup of $\Gamma$.

We use the symbol $\xi_{\Phi}$ to denote the characteristic function of $\Phi$.

If $A$ is a Banach algebra, an $A$-module is a (left) module $K$ over $A$ which is also a Banach space, and such that $\|f * k\| \leqq\|f\|\|k\|$ for all $f \in A, k \in K$ (* denoting the module composition). In particular we shall consider $L_{\Gamma}$ modules. An element $k$ of an $L_{\Gamma}$ module $K$ is called factorable if there exist $f \in L_{\Gamma}$ and $k^{\prime} \in K$ such that $k=f * k^{\prime}$. The factorable elements of $K$ form a closed submodule $K_{\text {abs }}$ of $K$ [5]. $K$ is said to be absolutely continuous if $K_{\mathrm{abs}}=K$. For instance, $L_{\Gamma}$ is an absolutely continuous $L_{\Gamma}$ module. It follows that $\left(K_{\mathrm{abs}}\right)_{\mathrm{abs}}=K_{\mathrm{abs}}$ for every $K$. It is clear that $\lim _{i} u_{i} * k=k$ for every $k \in K_{\text {abs }}$ and every approximate identity $\left(u_{i}\right)_{i \in I}$ in $L_{\Gamma}$. For $\sigma \in \Gamma$ we define a norm-preserving left shift $k \rightarrow k_{\sigma}$ in $K_{\text {abs }}$ by

$$
\left(f * k^{\prime}\right)_{\sigma}=f_{\sigma} * k^{\prime}, \quad f \in L_{\Gamma}, k^{\prime} \in K
$$

(see [5]). Then

$$
f * k_{\sigma}=f^{\sigma} * k, \quad f \in L_{\Gamma}, k \in K_{\mathrm{abs}}, \sigma \in \Gamma .
$$

For every $k \in K_{\mathrm{abs}}, k_{\sigma}$ depends continuously on $\sigma$. For all $f \in L_{\Gamma}, k \in K_{\mathrm{abs}}$ and $k^{*} \in K^{*}$, we have by [5]

$$
k^{*}(f * k)=\int_{\Gamma} f(\sigma) k^{*}\left(k_{\sigma^{-1}}\right) d \sigma .
$$

3. Reduction to order-free modules. Let $K$ be an $L_{\Gamma}$ module. We call $K$ order-free if there is no $k \in K$ such that $k \neq 0$ and such that $f * k=0$ for every $f \in L_{\Gamma}$. Every absolutely continuous module is order-free, because if $K$ is such a module, then for all $k \in K, k \in$ closure $\left\{f * k: f \in L_{\Gamma}\right\}$.

In general, for an $L_{\Gamma}$ module $K$ we call $\left\{k \in K: f * k=0\right.$ for every $\left.f \in L_{\Gamma}\right\}$ the order submodule $K_{0}$ of $K$. Note that $k \in K$ lies already in $K_{0}$ if there is a measurable $\Phi \subseteq \Gamma$, not locally null, such that $L_{\Phi} * k=\{0\}$. (Then for every $\sigma \in \Gamma,\{0\}=\left(L_{\Phi} * k\right)_{\sigma^{-1}}$ $=\left(L_{\Phi}\right)_{\sigma^{-1}} * k=\left(L_{\sigma \Phi}\right) * k$, so that, by $(2.2), L_{\Gamma} * k=\mathrm{Cl}\left(\sum_{\sigma \in \Gamma} L_{\sigma \Phi}\right) * k=\{0\}$.) The 
Banach space $K / K_{0}$ is made into an $L_{\Gamma}$ module by the definition

$$
f *\left(k+K_{0}\right)=(f * k)+K_{0} \quad\left(f \in L_{\Gamma}, k \in K\right) .
$$

This $K / K_{0}$ is always order-free.

Most modules we shall deal with are order-free, e.g., $C_{\infty}(X), M(X)$ and $L^{p}(X)$ $(1 \leqq p \leqq \infty)$. However, modules with order sometimes arise in a natural way. Thus, if $K$ is an $L_{\Gamma}$ module, we may define a module composition on $K^{*}$ by the following definition.

3.1. Definition. $\left(f * k^{*}\right)(k)=k^{*}\left(f^{\prime} * k\right),\left(f \in L_{\Gamma}, k \in K, k^{*} \in K^{*}\right)$.

$K^{*}$ is then an $L_{\Gamma}$ module. It is not hard to prove that $K^{*}$ is order-free if and only if $K$ is absolutely continuous. In fact, there is a natural isometrical module homomorphism of $\left(K_{\mathrm{abs}}\right) *$ onto $K^{*} /\left(K^{*}\right)_{0}$.

Let $\Omega$ be a measurable subset of $\Gamma$ that is not locally null, and assume that $L_{\Omega}$ is a subalgebra of $L_{\Gamma}$. Let $K$ be an $L_{\Gamma}$ module.

3.2. Definition. A continuous linear map $R: L_{\Omega} \rightarrow K$ is an $\left(L_{\Omega}, K\right)$-homomorphism if $R(f * g)=f * R g\left(f, g \in L_{\Omega}\right)$. The collection of $\left(L_{\Omega}, K\right)$-homomorphisms we denote by $\Re_{\Omega}(K)$. When $\Omega=\Gamma$ we suppress the $\Omega$ and write $\Re(K)$.

3.3. TheOREM. For any $L_{\Gamma}$ module $K, \Re_{\Omega}(K)$ is the direct sum of $\Re_{\Omega}\left(K_{\mathrm{abs}}\right)$ and $\Re_{\Omega}\left(K_{0}\right)$. In particular, in case $K$ is order-free, then $R(f) \in K_{\mathrm{abs}}$ for all $R \in \Re_{\Omega}(K)$ and $f \in L_{\Omega}$.

Proof. If $k \in K_{\text {abs }}$, then $\lim _{i} u_{i} * k=k$ for every approximate identity $\left(u_{i}\right)_{i \in I}$ in $L_{\Gamma}$; therefore $k \notin K_{0}$ if $k \neq 0$. Then by the definitions of $\Re_{\Omega}\left(K_{\mathrm{abs}}\right)$ and $\Re_{\Omega}\left(K_{0}\right)$, their intersection is $\{0\}$. Thus we need only show that $\Re_{\Omega}(K)=\Re_{\Omega}\left(K_{\mathrm{abs}}\right)+\Re_{\Omega}\left(K_{0}\right)$.

Let $R \in \Re_{\Omega}(K)$ be given. Take $\sigma \in d \Omega$. As we mentioned in $\S 2$ there exists an approximate identity $\left(u_{i}\right)_{i \in I}$ in $L_{\Gamma}$ such that for each $i \in I,\left(u_{i}\right)^{\sigma^{-1}} \in L_{\Omega}$. Then $f \in L_{\Omega}$ implies that $f_{\sigma^{-1}} \in L_{\Omega}$ and

$$
\begin{aligned}
R\left(f_{\sigma^{-1}}\right) & =\lim _{i} R\left(u_{i} * f_{\sigma^{-1}}\right)=\lim _{i} R\left(\left(u_{i}\right)^{\sigma^{-1}} * f\right) \\
& =\lim _{i}\left[\left(u_{i}\right)^{\sigma^{-1}} * R f\right] .
\end{aligned}
$$

Since $\left(u_{i}\right)^{\sigma^{-1}} * R f \in K_{\mathrm{abs}}$ and $K_{\mathrm{abs}}$ is closed in $K$, it follows that $R\left(f_{\sigma^{-1}}\right) \in K_{\mathrm{abs}}$. We define $R_{a}: L_{\Omega} \rightarrow K$ by $R_{a}(f)=\left[R\left(f_{\sigma}^{-1}\right)\right]_{\sigma}$. Then $R_{a}$ is a continuous linear map of $L_{\Omega}$ into $K_{\text {abs }}$. Take $g \in L_{\Omega}$. For $f \in L_{\Omega \sigma}$ we have $f^{\sigma} \in L_{\Omega}$, so that $f * R_{a} g=f^{\sigma} * R\left(g_{\sigma^{-1}}\right)$ $=R\left(f^{\sigma} * g_{\sigma^{-1}}\right)=R(f * g)=f * R g$. Thus $L_{\Omega \sigma} *\left(R g-R_{a} g\right)=\{0\}$. It follows that $R g-R_{a} g \in K_{0}$, and we conclude that $R-R_{a}$ is a continuous linear map $L_{\Omega} \rightarrow K_{0}$. Moreover, for all $f, g \in L_{\Omega}$ we obtain

$$
\begin{aligned}
f * R_{a} g & =f * R g=\left[(f * R g)_{\sigma^{-1}}\right]_{\sigma}=\left(f_{\sigma^{-1}} * R g\right)_{\sigma} \\
& =\left[R\left(f_{\sigma}^{-1} * g\right)\right]_{\sigma}=\left[R\left((f * g)_{\sigma^{-1}}\right)\right]_{\sigma} \\
& =R_{a}(f * g) .
\end{aligned}
$$


Thus $R_{a}$ is a module homomorphism, and consequently, so is $R-R_{a}$. We obtain $R_{a} \in \Re_{\Omega}\left(K_{\mathrm{abs}}\right), R-R_{a} \in \Re_{\Omega}\left(K_{0}\right)$.

The elements of $\Re_{\Omega}\left(K_{\mathrm{abs}}\right)$ can be characterized in terms of the shift in $K_{\mathrm{abs}}$.

3.4. Theorem. A continuous linear map $R: L_{\Omega} \rightarrow K_{\mathrm{abs}}$ is in $\Re_{\Omega}\left(K_{\mathrm{abs}}\right)$ if and only if $R\left(f_{\sigma^{-1}}\right)=(R f)_{\sigma^{-1}}$ for all $f \in L_{\Omega}$ and $\sigma \in d \Omega$.

Proof. Take $R \in \Re_{\Omega}\left(K_{\mathrm{abs}}\right), \sigma \in d \Omega$. By the proof of the preceding theorem, $R f-\left[R\left(f_{\sigma^{-1}}\right)\right]_{\sigma} \in K_{0}$ for every $f \in L_{\Omega}$. But of course $R f-\left[R\left(f_{\sigma^{-1}}\right)\right]_{\sigma} \in K_{\mathrm{abs}}$. Therefore $R f=\left[R\left(f_{\sigma^{-1}}\right)\right]_{\sigma}$. In other words, $(R f)_{\sigma^{-1}}=R\left(f_{\sigma^{-1}}\right)$.

Conversely, let $R: L_{\Omega} \rightarrow K_{\text {abs }}$ be a linear continuous map such that $R\left(f_{\sigma^{-1}}\right)$ $=(R f)_{\sigma^{-1}}$ for all $f \in L_{\Omega}$ and $\sigma \in d \Omega$. For any $f, g \in L_{\Omega}$ and any $k^{*} \in K^{*}$ we have by (2.5) that

$$
\begin{aligned}
k^{*}(f * R g) & =\int_{\Gamma} f(\sigma) k^{*}\left((R g)_{\sigma}-1\right) d \sigma \\
& =\int_{\Gamma} f(\sigma)\left\{\left[R^{*}\left(k^{*}\right)\right]\left(g_{\sigma^{-1}}\right)\right\} d \sigma \\
& =\left[R^{*}\left(k^{*}\right)\right](f * g)=k^{*}(R(f * g)),
\end{aligned}
$$

so that $f * R g=R(f * g)$. Thus $R \in \Re_{\Omega}\left(K_{\text {abs }}\right)$.

In order to describe $\Re_{\Omega}\left(K_{0}\right)$ we need a little more knowledge about the algebra $L_{\Omega}$. There exists an open set $T \subset \Gamma$ such that $L_{T}$ is the closed linear span of $\left\{f * g: f, g\right.$ in $\left.L_{\Omega}\right\}$. The proof of this statement and the explicit definition of $T$ are in [5]. (Note that $\mathscr{T}=\mathcal{O}$ because $X=\Gamma$.) In terms of this set $T$ we have a simple characterization of $\left(L_{\Omega}, K_{0}\right)$-homomorphisms.

3.5. TheOREM. A continuous linear map $R: L_{\Omega} \rightarrow K_{0}$ is in $\Re_{\Omega}\left(K_{0}\right)$ if and only if $R=0$ on $L_{T}$.

Proof. If $R=0$ on $L_{T}$, then $R(f * g)=0$ for all $f, g \in L_{\Omega}$. On the other hand, $L_{\Omega} * K_{0}=\{0\}$ and $f * R g \in L_{\Omega} * K_{0}$. Thus $f * R g=0$ and $R \in \Re_{\Omega}\left(K_{0}\right)$. Conversely, for $R \in \Re_{\Omega}\left(K_{0}\right)$ and $f, g \in L_{\Omega}$ we have $R(f * g)=f * R g \in L_{\Omega} * K_{0}=\{0\}$. Since $R$ is linear and continuous, $R(h)=0$ for all $h \in L_{T}$.

We mention that $\Re_{\Omega}\left(K_{0}\right)=\{0\}$ if $L_{T}=L_{\Omega}$. In particular, $L_{T}=L_{\Omega}$ if $1 \in d \Omega$, because then $L_{\Omega}$ contains an approximate identity of $L_{\Gamma}$. To wit, if $\Omega=\Gamma$ we have $\Re\left(K_{0}\right)=\{0\}$.

4. A decomposition theorem for module homomorphisms. Let $\Omega$ be a semigroup. An equivalence relation $\sim$ in $\Omega$ is called an "ideal equivalence relation" if $\sigma \tau \sim \tau$ for all $\sigma, \tau \in \Omega$. Let us define the equivalence relation $\approx$ in $\Omega$ by $\sigma \approx \tau$ if and only if $\sigma \sim \tau$ for every ideal equivalence relation $\sim$ in $\Omega$. Then $\approx$ is itself an ideal equivalence relation. Among all ideal equivalence relations $\approx$ is the finest, has the smallest equivalence classes. Explicitly, $\sigma \approx \tau$ if and only if there exists a finite sequence $\sigma=\sigma_{1}, \sigma_{2}, \ldots, \sigma_{n}=\tau$ in $\Omega$ such that $\left(\Omega \sigma_{i}\right) \cap\left(\Omega \sigma_{i+1}\right) \neq \varnothing$ for each $i$. 
The equivalence classes modulo $\approx$ form a set $\mathscr{J}(\Omega)$. If $\Omega$ is abelian or contains a right unit, then $\mathscr{J}(\Omega)$ consists of only one element; indeed, if $\Omega$ is abelian, then $\sigma \approx \tau \sigma=\sigma \tau \approx \tau$ for all $\sigma, \tau \in \Omega$, and if $\Omega$ has a right unit $\eta$, then $\sigma=\sigma \eta \approx \eta$ for all $\sigma \in \Omega$. If $\sigma \in J \in \mathscr{J}(\Omega)$ and if $\tau \in \Omega$, then $\left(\tau^{2}\right) \sigma=\tau(\tau \sigma)$, so $\Omega J \subseteq J$, which means that $J$ is a left ideal of $\Omega$.

4.1. Lemma. Let $\Omega$ be a measurable subsemigroup of $\Gamma$, with $\Omega$ not locally null. Then there exists a neighborhood $\Phi$ of $1 \in \Gamma$ such that $\Omega \cap \Phi J \subseteq J$ for every $J \in \mathscr{J}(\Omega)$. In particular, the ideal equivalence classes are relatively open in $\Omega$, and $f=\sum\left\{f \xi_{J}: J \in \mathscr{J}(\Omega)\right\}$ for all $f \in L_{\Omega}$. Furthermore, $\sigma^{-1} J \cap \Omega=J$ for all $\sigma \in \Omega$ and $J \in \mathscr{J}(\Omega)$.

Proof. Inasmuch as $\Omega \Omega$ contains a nonempty open subset of $\Gamma$ by [6, 20.17], there is a $\beta \in \Omega$ and a neighborhood $\Phi$ of 1 such that $\beta \Phi \subseteq \Omega$. Let $\sigma \in \Omega$. For all $\tau \in \Omega \cap \Phi \sigma$ we have $\beta \tau \sigma^{-1} \in \beta \Phi \subseteq \Omega$ and consequently $\sigma \approx\left(\beta \tau \sigma^{-1}\right) \sigma=\beta \tau \approx \tau$. Thus, if $\sigma \in J \in \mathscr{J}(\Omega)$, then $\Omega \cap \Phi \sigma \subseteq J$, so that $\Omega \cap \Phi J \subseteq J$. To prove the last statement, we notice that if $\tau \in J, \beta \in \Omega$ and $\sigma \beta \in J$, then $\tau \approx \sigma \beta \approx \beta$; therefore $\sigma^{-1} J \cap \Omega \subseteq J$, while the converse inclusion is obvious.

4.2. Corollary. Let $\Omega$ be as above. If $\Omega$ is connected, then $\mathscr{J}(\Omega)$ contains only one element.

Let $\Omega$ be a measurable subset of $\Gamma$. It is known that if $L_{\Omega}$ is an algebra, then $d \Omega$ is a closed subsemigroup of $\Gamma$. Furthermore, for any $\sigma \in d \Omega$, we have $\sigma \Omega \subseteq \Omega$ l.a.e. and $\Omega \sigma \subseteq \Omega$ 1.a.e. (see $\S 2$ ). Inasmuch as every $J \in \mathscr{J}(d \Omega)$ is a subsemigroup, $L_{J}$ and $L_{\Omega \cap J}$ are nontrivial algebras, the latter because $J$ is a nonempty relatively open subset of $d \Omega$.

We have sufficient machinery to decompose $\left(L_{\Omega}, K\right)$-homomorphisms.

4.3. Theorem. Let $\Omega$ be a measurable subset of $\Gamma$ that is not locally null. Assume $L_{\Omega}$ is an algebra, and $K$ an $L_{\Gamma}$ module which is order-free. For every $R \in \Re_{\Omega}(K)$ there is a family $\left\{R_{J}: J \in \mathscr{J}(d \Omega)\right\}$ of elements of $\Re(K)$, such that

$$
R(f)=\sum_{J \in \mathscr{J}(d \Omega)} R_{J}\left(f \xi_{J}\right), \quad f \in L_{\Omega} .
$$

Furthermore, $\|R\|=\sup \left\{\left\|R_{J}\right\|: J \in \mathscr{J}(d \Omega)\right\}$.

Conversely, for every norm-bounded family $\left\{R_{J}: J \in \mathscr{J}(d \Omega)\right\}$ in $\Re(K)$ the equation $\left(^{*}\right)$ defines an $R \in \Re_{\Omega}(K)$.

Proof. Let $R \in \Re_{\Omega}(K)$ be given to us. Let $\alpha \in d \Omega$, and let $\left(u_{i}\right)_{i \in I}$ be an approximate identity in $L_{\Gamma}$ with norm 1 and such that $\left(u_{i}\right)_{\alpha}-1 \in L_{\Omega}$ for every $i$. Next let $\sigma \in \Gamma$ and $f \in L_{\sigma \Omega}$. Then $f_{\sigma} \in L_{\Omega}$ and hence $\left(f_{\sigma}\right)^{\alpha^{-1}} \in L_{\Omega \alpha} \subseteq L_{\Omega}$. Thus

$$
\begin{aligned}
\left\{R\left[\left(f_{\sigma}\right)^{\alpha^{-1}}\right]\right\}_{\sigma^{-1}} & =\lim _{i}\left\{R\left[\left(f_{\sigma}\right)^{\alpha^{-1}} * u_{i}\right]\right\}_{\sigma^{-1}} \\
& =\lim _{i}\left\{R\left[f_{\sigma} *\left(u_{i}\right)_{\alpha^{-1}}\right]\right\}_{\sigma^{-1}} \\
& =\lim _{i}\left\{f_{\sigma} * R\left[\left(u_{i}\right)_{\alpha^{-1}}\right]\right\}_{\sigma^{-1}}=\lim _{i} f * R\left[\left(u_{i}\right)_{\alpha^{-1}}\right] .
\end{aligned}
$$


In particular, the latter limit exists for any $\sigma \in \Gamma$ and $f \in L_{\sigma \Omega}$. Since the approximate identity is bounded, so is $\left\{R\left[\left(u_{i}\right)_{\alpha}^{-1}\right]: i \in I\right\}$, which means that if $D$ is defined as $\left\{f \in L_{\Gamma}: \lim _{i}\left[f * R\left(u_{i}\right)_{\alpha^{-1}}\right]\right.$ exists $\}$, then $D$ must be a closed linear subspace of $L_{\Gamma}$, and by the calculation above, $D \supseteq \bigcup_{\sigma \in \Gamma} L_{\sigma \Omega}$. Thus $D \supseteq \mathrm{Cl}\left(\sum_{\sigma \in \Gamma} L_{\sigma \Omega}\right)=L_{\Gamma}$ (see 2.2). Hence we can define $R_{\alpha}: L_{\Gamma} \rightarrow K$ by

$$
R_{\alpha}(f)=\lim _{i} f^{\alpha} * R\left(\left(u_{i}\right)_{\alpha^{-1}}\right), \quad f \in L_{\Gamma}
$$

Since $f, g \in L_{\Gamma}$ means that $(f * g)^{\alpha}=f * g^{\alpha}$, evidently $R_{\alpha} \in \Re(K)$. Recapitulating, we have taken an $R \in \Re_{\Omega}(K)$, so that $R$ is defined only on $L_{\Omega}$, and from it we have defined a module homomorphism $R_{\alpha}$ on the whole of $L_{\Gamma}$. The particular $R_{\alpha}$ we obtain depends (or at least appears to depend) upon the $\alpha \in d \Omega$ picked at the beginning of the proof. In any case, we next show that $R_{\alpha}$ is an extension of $R$ restricted to $\Omega \cap J$, where $\alpha \in J$.

It is easy to show that $R_{\alpha}=R$ on $L_{\Omega \alpha}$. After all, if $f \in L_{\Omega \alpha}$ then $f^{\alpha} \in L_{\Omega}$ and $R_{\alpha}(f)=\lim _{i} R\left(f^{\alpha} *\left(u_{i}\right)_{\alpha}-1\right)=\lim _{i} R\left(f * u_{i}\right)=R(f)$. Now we determine an ideal equivalence relation on $d \Omega$. Write $\beta \sim \sigma$ if $R_{\beta}=R_{\sigma}$. Let $\beta, \sigma \in d \Omega$. We must show that $R_{\sigma \beta}=R_{\beta}$. On $L_{\Omega \beta}, R_{\beta}=R$ while on $L_{\Omega \sigma \beta}, R_{\sigma \beta}=R$. Since $\Omega \sigma \subseteq \Omega$ l.a.e., we have $L_{\Omega \sigma \beta} \subseteq L_{\Omega \beta}$ and $R_{\sigma \beta}=R=R_{\beta}$ on $L_{\Omega \sigma \beta}$. But $R_{\sigma \beta}$ and $R_{\beta}$ are module homomorphisms on $L_{\Gamma}$, so by Theorem 3.4 they are left translation invariant by any element of $\Gamma$. This means that they agree not only on $L_{\Omega \sigma \beta}$ but on $L_{\tau \Omega \sigma \beta}$ for any $\tau \in \Gamma$. Hence $R_{\sigma \beta}=R_{\beta}$ on $\mathrm{Cl}\left(\sum_{\tau \in \Gamma} L_{\tau \Omega \sigma \beta}\right)=L_{\Gamma}$ (see 2.2). This proves that $R_{\sigma \beta}=R_{\beta}$ and $\sim$ is an ideal equivalence relation. Next, if $\alpha \in d \Omega$, then there is a $J \in \mathscr{J}(d \Omega)$ such that $\alpha \in J$. For any $\beta \in J, R_{\alpha}=R_{\beta}$, so that we may define $R_{J}$ as $R_{\alpha}$ and take away the apparent dependence on the particular $\alpha \in J$. Then $R_{J}=R$ on $\mathrm{Cl}\left(\sum_{\beta \in J} L_{\Omega \beta}\right)$. We note that $J$ is a closed subset of $d \Omega$, since $d \Omega \mid J$ is relatively open in $d \Omega$ by Lemma 4.1 (where $\Omega$ is replaced by $d \Omega$ ). Because $d \Omega$ is closed in $\Gamma$, we know that $J$ is also closed in $\Gamma$, so $J \supseteq d J$. Take $\beta \in d \Omega$. Then $R_{J}=R$ on

$$
\mathrm{Cl}\left(\sum_{\sigma \in J} L_{\Omega \sigma}\right) \supseteq \mathrm{Cl}\left(\sum_{\sigma \in d J} L_{\Omega \sigma}\right)=\sum_{\tau \in d \Omega} L_{\tau J} \supseteq L_{\beta(\Omega \cap J)} \quad \text { (by 2.1), }
$$

which is just perfect for us because if $f \in L_{\Omega \cap J}$ then $f_{\beta}-1 \in L_{\beta(\Omega \cap J)}$ and consequently $R_{J}(f)=\left[R_{J}\left(f_{\beta}-1\right)\right]_{\beta}=\left[R\left(f_{\beta}-1\right)\right]_{\beta}=R(f)$ by the translation invariance of $R$. Thus $R=R_{J}$ on $L_{\Omega \cap J}$. We have thus shown that $R$ yields the module homomorphism $R_{J}$ defined on all of $L_{\Gamma}$ in such a way that $R$ and $R_{J}$ agree on $\Omega \cap J$. From Lemma 4.1 we infer that for any $f \in L_{\Omega}=L_{\Omega \cap d \Omega}, f=\sum_{J}\left\{f \xi_{J}\right\}$, with the result that $R(f)=\sum_{J}\left\{R\left(f \xi_{J}\right)\right\}=\sum_{J}\left\{R_{J}\left(f \xi_{J}\right)\right\}$, which proves $\left(^{*}\right)$. As for the norm inequalities, $\left\|R_{\alpha}\right\| \leqq\|R\|$ since the approximate identity is bounded by 1 . Thus $\left\|R_{J}\right\| \leqq\|R\|$ for every $J \in \mathscr{J}(d \Omega)$. The inequality $\|R\| \leqq \sup \left\{\left\|R_{J}\right\|: J \in \mathscr{J}(d \Omega)\right\}$ follows from (*). Hence $\|R\|=\sup \left\{\left\|R_{J}\right\|: J \in \mathscr{J}(d \Omega)\right\}$.

We have yet to prove the converse. Let $\left\{R_{J}: J \in \mathscr{J}(d \Omega)\right\}$ be a family of elements of $\Re(K)$ such that $\left\{\left\|R_{J}\right\|: J \in \mathscr{J}(d \Omega)\right\}$ is bounded. Then $\left(^{*}\right)$ defines a continuous linear map $R: L_{\Omega} \rightarrow K$. Now let $\sigma \in d \Omega$. By the last part of Lemma 4.1, 
$\sigma^{-1} J \cap d \Omega=J$ for every $J \in \mathscr{J}(d \Omega)$. This means that for all $f \in L_{\Omega}$,

Thus

$$
f_{\sigma^{-1}} \xi_{J}=\left(f \xi_{\sigma^{-1}}\right)_{\sigma^{-1}}=\left(f \xi_{d \Omega} \xi_{\sigma^{-1}}\right)_{\sigma^{-1}}=\left(f \xi_{J}\right)_{\sigma^{-1}}
$$

$$
\begin{aligned}
R\left(f_{\sigma^{-1}}\right) & =\sum_{J} R_{J}\left(f_{\sigma^{-1}} \xi_{J}\right)=\sum_{J} R_{J}\left(\left(f \xi_{J}\right)_{\sigma^{-1}}\right) \\
& =\left(\sum_{J} R_{J}\left(f \xi_{J}\right)\right)_{\sigma^{-1}}=(R(f))_{\sigma^{-1}},
\end{aligned}
$$

so by Theorem $3.4, R$ is an $\left(L_{\Omega}, K\right)$-module homomorphism.

The theorem says that to any module homomorphism $R$ defined on $L_{\Omega}$ there corresponds a collection $\left(R_{J}\right)_{J \in \mathscr{J}(d \Omega)}$ of module homomorphisms on $L_{\Gamma}$, and each $R_{J}$ is the unique extension of $R$ restricted to $\Omega \cap J$. Thus if we have complete knowledge of $\Re(K)$, then the problem of $\Re_{\Omega}(K)$ is completely solved as well. In other words, the problem of characterizing $\Re_{\Omega}(K)$ is reduced to the problem of characterizing $\Re(K)$. Besides what occurs in this paper, there is a discussion of $\Re(K)$ in [3], [8], and [9].

Sometimes Theorem 4.3 takes on a simpler form.

4.4. Corollary. Assume that $\Omega$ has at least one of the following properties:

$(\alpha) \Omega$ is commutative.

(B) $1 \in d \Omega$.

$(\gamma) \Omega$ is connected.

Then every $R \in \Re_{\Omega}(K)$ has a unique extension to an $\bar{R} \in \Re(K)$, and $\|\bar{R}\|=\|R\|$.

Proof. $\mathscr{J}(d \Omega)$ contains but one element.

It would be conceivable, no matter what $\Gamma$ and $\Omega \subseteq \Gamma$ are, that $\mathscr{J}(d \Omega)$ consisted of but one element. In fact it would be desirable, since then any module homomorphism from $L_{\Omega}$ to $K$ could be extended-uniquely-to a module homomorphism from $L_{\Gamma}$ to $K$. However, this is not the case. Let $\Gamma$ be the free group with two generators, $\alpha$ and $\beta$, and let $\Gamma$ have the discrete topology. Let $\Phi$ be the subsemigroup generated by $1, \alpha$, and $\beta$, and let $\Omega$ be the subsemigroup $\Phi_{\alpha} \cup \Phi \beta$. We define the continuous, linear map $R: L_{\Omega} \rightarrow L_{\Gamma}$ by

$$
R\left(\xi_{\{\sigma \alpha\}}\right)=\xi_{\{\sigma \alpha\}}, \quad \sigma \in \Phi, \quad R\left(\xi_{\{\sigma \beta\}}\right)=\xi_{\{\sigma \alpha\}}, \quad \sigma \in \Phi .
$$

Then $R \in \Re_{\Omega}\left(L_{\Gamma}\right)$, but there is no extension $\bar{R} \in \Re\left(L_{\Gamma}\right)$ such that $R$ and $\bar{R}$ coincide on $L_{\Omega}$.

From the definition of $\mathscr{J}(d \Omega)$ we see that $\Omega \cap d \Omega$ is partitioned by $\{\Omega \cap J: J \in \mathscr{J}(d \Omega)\}$. This means that $L_{\Omega}=L_{\Omega \cap d \Omega}=\sum_{J} L_{\Omega \cap J}$ where the $L_{\Omega \cap J}$ are pairwise disjoint (except for $\{0\}$ ) left ideals of $L_{\Omega}$. We prove below that $\left\{L_{\Omega \cap J}: J \in \mathscr{J}(d \Omega)\right\}$ forms the finest decomposition of $L_{\Omega}$ as the sum of disjoint left ideals of the form $L_{\theta}$.

4.5. THEOREM. Let $\left(\theta_{i}\right)_{i \in I}$ be a set of measurable subsets of $\Omega$ such that each $L_{\theta_{i}}$ is $a$ left ideal of $L_{\Omega}$ and such that $L_{\Omega}=\mathrm{Cl}\left(\sum_{i \in I} L_{\theta_{i}}\right)$ and $L_{\theta_{i}} \cap L_{\theta_{j}}=\{0\}$ for $i \neq j$. Then for each $i, L_{\theta_{i}}$ is the closure of $\sum\left\{L_{\Omega \cap J}: J \in \mathscr{J}(d \Omega), L_{\Omega \cap J} \subseteq L_{\theta_{i}}\right\}$. 
Proof. Let $P_{i}: L_{\Omega} \rightarrow L_{\theta_{i}}$ be defined by $P_{i}(f)=f \xi_{\theta_{i}}$. Then $P_{i}$ is continuous, linear, a projection from $L_{\Omega}$ onto $L_{\theta_{i}}$, and $f \in L_{\Omega}$ implies that $f=\sum_{i \in I} P_{i}(f)$. Fix $i \in I$. Let $f, g \in L_{\Omega}$. Since by assumption $L_{\theta_{i}}$ is a left ideal, and since $P_{i}(g) \in L_{\theta_{i}}$, we have $f *\left[P_{i}(g)\right] \in L_{\theta_{i}}$, and thus

$$
P_{i}(f * g)-f *\left[P_{i}(g)\right]=P_{i}(f * g)-P_{i}\left\{f *\left[P_{i}(g)\right]\right\}=P_{i}\left\{f *\left[g-P_{i}(g)\right]\right\} .
$$

But $g-\left[P_{i}(g)\right] \in L_{\Omega \mid \theta_{i}}=\mathrm{Cl}\left(\sum_{j \neq i} L_{\theta_{j}}\right)$, so

$$
f *\left(g-\left[P_{i}(g)\right]\right) \in \mathrm{Cl}\left(\sum_{j \neq i} f * L_{\theta}\right) \subseteq \mathrm{Cl}\left(\sum_{j \neq i} L_{\theta_{j}}\right)
$$

since each $L_{\theta_{i}}$ is a left ideal in $L_{\Omega}$. Thus $P_{i}\left(f *\left\{g-\left[P_{i}(g)\right]\right\}\right)=0$. Hence $P_{i} \in \Re_{\Omega}\left(L_{\Gamma}\right)$. Hence Theorem 4.3 applies, and there exists a collection $\left\{R_{J}: J \in \mathscr{J}(d \Omega)\right\} \subseteq \Re\left(L_{\Gamma}\right)$ such that $P_{i}(f)=\sum_{J} R_{J}\left(f \xi_{J}\right)$, for all $f \in L_{\Gamma}$. By a theorem of G. Wendel (see [10]), every $R_{J} \in \mathfrak{R}\left(L_{\Gamma}\right)$ determines a $\mu_{J} \in M(\Gamma)$ such that $R_{J}(g)=g * \mu_{J}$, for all $g \in L_{\Gamma}$. Then

$$
f \xi_{\theta_{i}}=P_{i}(f)=\sum_{J}\left(f \xi_{J}\right) * \mu_{J}, \quad \text { for all } f \in L_{\Omega} .
$$

Using this decomposition we show next that for each $J \in \mathscr{J}(d \Omega)$, either $\mu_{J}=0$ or $\mu_{J}=\delta_{1}$, the point mass at $1 \in \Gamma$. To that end, let $J \in \mathscr{J}(d \Omega)$ such that $\theta_{i} \cap J$ is not locally null. If $f \in L_{\theta_{i} \cap J}$ then the formula displayed above yields us $f=f * \mu_{\mathrm{J}}$. Thus $L_{\theta_{i} \cap J}=L_{\theta_{i} \cap J} * \mu_{J}$, so that $L_{\theta_{i} \cap J} *\left(\delta_{1}-\mu_{J}\right)=0$. Then $\delta_{1}-\mu_{J}$ lies in the order submodule of $M(\Gamma)$. However, $M(\Gamma)$ is order-free. Consequently $\delta_{1}-\mu_{J}=0$, or $\delta_{1}=\mu_{\mathrm{J}}$. On the other hand, if $J$ is such that $J \cap \theta_{i}$ is locally null, then by a similar reasoning, $\mu_{J}=0$. Finally, we note that $\left(f \xi_{J}\right) * \delta_{1}=f \xi_{J}$, while $\left(f \xi_{J}\right) * 0=0$, so that $f \in L_{\Omega}$ implies that

$$
f \xi_{\theta_{l}}=\sum\left\{f \xi_{J}: J \in \mathscr{J}(d \Omega), \mu_{J}=\delta_{1}\right\}
$$

Since every element of $L_{\theta_{i}}$ is of the form $f \xi_{\theta_{i}}$ for an appropriate $f \in L_{\Omega}$, we see that we have decomposed $L_{\theta_{i}}$ as hypothesized. (The closure appearing in the statement of the theorem merely preserves the widespread convention that the sum of a collection of spaces contains only finite sums of elements of the spaces involved.)

We remark that there may very well be finer decompositions of $L_{\Omega}$ into a sum of ideals not of the form $L_{\theta}$. Thus, if $\mu$ is an idempotent measure on $\Gamma$, then $L_{\Gamma}$ is the direct sum of $L_{\Gamma} * \mu$ and $L_{\Gamma} *\left(\delta_{1}-\mu\right)$, while $\mathscr{J}(\Gamma)$ contains only one element.

Let us see how $\$ \$ 3$ and 4 have simplified the problem of finding $\Re_{\Omega}(K)$-module homomorphisms for an arbitrary $L_{\Gamma}$ module $K$. In the first place, we found that $\Re_{\Omega}(K)=\Re_{\Omega}\left(K_{0}\right) \oplus \Re_{\Omega}\left(K_{\text {abs }}\right)$, in Theorem 3.3, and then described as completely as we will here the space $\Re_{\Omega}\left(K_{0}\right)$, which from Theorem 3.5 turns out to be the collection of linear, continuous maps from $L_{\Omega}$ to $K_{0}$ which map $L_{T}$ into 0 . That done, we directed our attention to those $K$ which were order-free, showing that $R \in \Re_{\Omega}\left(K_{\text {abs }}\right)$ meant that $R$ corresponded to a collection $\left\{R_{J}: R_{J} \in \mathfrak{A}\left(K_{\text {abs }}\right), J \in \mathscr{J}(d \Omega)\right\}$ (Theorem 
4.3). Consequently we know $\Re_{\Omega}\left(K_{\text {abs }}\right)$ provided we know $\Re\left(K_{\text {abs }}\right)$, which in this case is none other than $\Re(K)$ if $K$ is order-free, by Theorem 3.3. Later we will use these results in special examples.

5. Module homomorphisms on $L_{\Gamma}$. In this section we assume that $\Omega=\Gamma$. For any $L_{\Gamma}$ module $K$ whatsoever, we can determine a special subspace of $\Re(K)$ in the following way. For $k \in K$, let $T_{K} k \in \Re(K)$ be defined by $\left(T_{K} k\right)(f)=f * k\left(f \in L_{\Gamma}\right)$. Indeed $K$ is order-free if and only if $T_{K}$ is injective. Then we have

5.1. TheOREM. (i) The definition $(f * R)(g)=R(g * f)\left(f, g \in L_{\Gamma}, R \in \mathfrak{R}(K)\right)$ turns $\Re(K)$ into an order-free $L_{\Gamma}$ module, thereby rendering $T_{K}$ a module homomorphism of $K$ into $\Re(K)$.

(ii) If $K$ is absolutely continuous, $T_{K}$ is an isometry.

(iii) The restriction of $T_{K}$ to $K_{\mathrm{abs}}$ is an isometry of $K_{\mathrm{abs}}$ onto $\Re(K)_{\mathrm{abs}}$.

(iv) $T_{\Re(K)}$ is an isometry of $\Re(K)$ onto $\Re(\Re(K))$.

Proof. (i) Except for showing that $\mathfrak{R}(K)$ is order-free the proof is a straightforward calculation. But if $L_{\Gamma} * R=\{0\}$, then $R\left(L_{\Gamma}\right)=R\left(L_{\Gamma} * L_{\Gamma}\right)=L_{\Gamma} * R\left(L_{\Gamma}\right)=\{0\}$, so $R=0$. To prove (ii), we note first that if $K$ is absolutely continuous, then $K$ is order-free, so $T_{K}$ is injective. Next, if $\left(u_{i}\right)_{i \in I}$ is an approximate identity of norm 1 in $L_{\Gamma}$ and if $k \in K$, then

$$
\begin{aligned}
\left\|T_{K} k\right\| & =\sup \left\{\|f * k\|: f \in L_{\Gamma} ;\|f\|_{1} \leqq 1\right\} \\
& \geqq \lim _{i}\left\|u_{i} * k\right\|=\|k\| .
\end{aligned}
$$

On the other hand, since $K$ is a Banach module, $\left\|T_{K} k\right\| \leqq\|k\|$. Now we prove (iii). The isometry we already have. Since $K_{\mathrm{abs}}$ is absolutely continuous, $T_{K} k$ is factorable in $\Re(K)$ for every $k \in K_{\text {abs }}$. Thus $T_{K}$ maps $K_{\text {abs }}$ into $\Re(K)_{\text {abs }}$. To show the map restricted to $K_{\text {abs }}$ is onto $\Re(K)_{\text {abs }}$, let $R \in \Re(K)_{\text {abs }}$. Then there exist $f \in L_{\Gamma}$ and $R^{\prime} \in \Re(K)$ such that $R=f * R^{\prime}$. Thus for all $g \in L_{\Gamma}$,

$$
\left\{T_{K}\left[R^{\prime}(f)\right]\right\}(g)=g *\left(R^{\prime} f\right)=\left(f * R^{\prime}\right)(g)=R(g) .
$$

Consequently, $R=T_{K}\left(R^{\prime}(f)\right)$. Since $L_{\Gamma}$ is factorable, there are $f_{1}, f_{2} \in L_{\Gamma}$ such that $f=f_{1} * f_{2}$. Then $R=T_{K}\left(R^{\prime}\left(f_{1} * f_{2}\right)\right)=T_{K}\left(f_{1} * R^{\prime}\left(f_{2}\right)\right) \in T_{K}\left(K_{\mathrm{abs}}\right)$, which is what we needed to prove. Finally we prove (iv), which is simple now. We have natural surjective isometries

$$
\Re(K) \rightarrow \Re\left(K_{\mathrm{abs}}\right) \rightarrow \Re\left(\Re(K)_{\text {abs }}\right) \rightarrow \Re(\Re(K)),
$$

the middle one coming from (iii) and the outer two by the comment following Theorem 3.4. The composition of these isometries is just $T_{\Re(K)}$.

5.2. TheOrem. Let Kbe an $L_{\Gamma}$ module. Every $k^{*} \in K_{\mathrm{abs}}{ }^{*}$ determines a $Q k^{*} \in \Re\left(K^{*}\right)$ by

$$
\left[\left(Q k^{*}\right) f\right](k)=k^{*}\left(f^{\prime} * k\right) \quad\left(f \in L_{\Gamma}, k \in K\right) .
$$


(If $K=K_{\mathrm{abs}}$, then $Q=T_{K^{*}}$ ) The map $Q$ defined by this equation is an isometric module homomorphism of $K_{\mathrm{abs}}$ * onto $\Re\left(K^{*}\right)$.

Proof. Certainly $Q k^{*}$ is linear and continuous as a map from $L_{\Gamma}$ to $K^{*}$. Also $\left\|Q k^{*}\right\| \leqq\left\|k^{*}\right\|$. Once one remembers that $(f * g)^{\prime}=g^{\prime} * f^{\prime}$, there is no trouble in showing that $Q k^{*} \in \mathfrak{R}\left(K^{*}\right)$. Then $Q$ is a linear map $K_{\text {abs }}{ }^{*} \rightarrow \mathfrak{R}\left(K^{*}\right)$ and $\|Q\| \leqq 1$. The proof that $Q$ is a module homomorphism is straightforward. Because the domain of $Q$ is $K_{\text {abs }}{ }^{*}, Q k^{*}=0$ only if $k^{*}=0$. Thus $Q$ is injective and we are done if we show it is surjective and $\left\|Q k^{*}\right\| \geqq\left\|k^{*}\right\|$ for all $k^{*} \in K_{\text {abs }}{ }^{*}$. Let $R \in \Re\left(K^{*}\right)$, and let $\left(u_{i}\right)_{i \in I}$ be an approximate identity in $L_{\Gamma}$, with $\left\|u_{i}\right\| \leqq 1$ for each $i$. If $f \in L_{\Gamma}$ and $j \in K$, then

$$
\begin{aligned}
(R f)(j) & =\lim _{i}\left(R\left(f * u_{i}\right)\right)(j) \\
& =\lim _{i}\left(f * R u_{i}\right)(j)=\lim _{i}\left(R u_{i}\right)\left(f^{\prime} * j\right) .
\end{aligned}
$$

Thus $\lim _{i}\left(R u_{i}\right)(k)$ exists for every $k \in K_{\text {abs }}$ and

$$
\left\|\lim _{i}\left(R u_{i}\right)(k)\right\| \leqq \sup _{i}\|R\|\left\|u_{i}\right\|\|k\| \leqq\|R\|\|k\| .
$$

Therefore we can define $k^{*} \in K_{\mathrm{abs}} *$ by $k^{*}(k)=\lim _{i}\left(R u_{i}\right)(k)$, for $k \in K_{\mathrm{abs}}$, with the result that $\left\|k^{*}\right\| \leqq\|R\|$. Now by the existence proof of $\lim _{i}\left(R u_{i}\right)(k)$ we have that for all $f \in L_{\Gamma}$ and $j \in K, \quad(R f)(j)=\lim _{i}\left(R u_{i}\right)\left(f^{\prime} * j\right)=k^{*}\left(f^{\prime} * j\right)=\left[\left(Q k^{*}\right) f\right](j)$. Thus $R=Q k^{*}$ and $\left\|Q k^{*}\right\| \geqq\left\|k^{*}\right\|$. This finishes the proof of the theorem.

With the aid of Theorems 5.1 and 5.2 we can compute $\Re(K)$ for most of the modules described in [5]. First, assume that $X$ is a locally compact space, and $\Gamma$ a group of homeomorphisms of $X$ such that the map $(\sigma, x) \rightarrow \sigma x(\sigma \in \Gamma, x \in X)$ is jointly continuous. Let $C_{X}$ be the Banach space of all continuous functions $k$ on $X$ such that for every $\varepsilon>0$ the set $\{x \in X:|k(x)| \geqq \varepsilon\}$ is compact. Then $C_{X}$ is an $L_{\Gamma}$ module with the module composition defined by

$$
f * k(x)=\int_{\Gamma} f(\sigma) k\left(\sigma^{-1} x\right) d \sigma, \quad x \in X,
$$

for $f \in L_{\Gamma}, k \in C_{X}$. (For details, see [4], [5].) We can make $M(X)$ an $L_{\Gamma}$ module by noting that it is the dual of $C_{X}$. Definition 3.1 yields

$$
(f * \mu) k=\mu\left(f^{\prime} * k\right), \quad f \in L_{\Gamma}, \mu \in M(X), k \in C_{X},
$$

and it turns out that $(f * \mu)(k)=\int_{X} \int_{\Gamma} k(\sigma x) f(\sigma) d \sigma d \mu(x)$.

Since $C_{X}$ is absolutely continuous, Theorem 5.2 tells us that $\Re(M(X))$ is canonically isomorphic to $M(X)$. (Unfortunately we have not been able to obtain a description of $\Re\left(C_{X}\right)$ itself!)

Now let $X$ possess a quasi-invariant measure $m_{X}$. We denote by $L_{X}^{p}(1 \leqq p \leqq \infty)$ the space usually called $L^{p}(X)$ or $L_{p}(X)$, and write $L_{X}$ instead of $L^{1}(X)$. The natural embedding $L_{X} \rightarrow M(X)$ makes $L_{X}$ a submodule of $M(X)$ (see [4]), and therefore induces an embedding $\Re\left(L_{X}\right) \rightarrow \Re(M(X))=M(X)$. The image of $\Re\left(L_{X}\right)$ in $M(X)$ is 
$N=\left\{\mu \in M(X):\right.$ for every $f \in L_{\Gamma}, f * \mu$ is absolutely continuous with respect to $\left.m_{X}\right\}$. This space has been investigated in [4], and in particular several conditions equivalent to $N$ equaling $M(X)$ appear there.

Since $L_{X}^{\infty}=\left(L_{X}\right)^{*}$ we can use (3.1) to make an $L_{\Gamma}$ module out of $L_{X}^{\infty}$. It turns out that for $f \in L_{\Gamma}$ and $k \in L_{X}^{\infty}$,

$$
f * k(x)=\int_{\Gamma} f(\sigma) k\left(\sigma^{-1} x\right) d \sigma, \quad \text { 1.a.e. } x \in X .
$$

We know $L_{X}$ is itself absolutely continuous; hence, again by Theorem $5.2, \Re\left(L_{X}^{\infty}\right)$ is isomorphic to $L_{X}^{\infty}$.

For $1 \leqq p \leqq \infty$, in [4], we introduced convolution products $L_{\Gamma} \times L_{X}^{p} \rightarrow L_{X}^{p}$, of which the module operations on $L_{X}$ and $L_{X}^{\infty}$, mentioned above, are special cases. For $1 \leqq p<\infty, L_{X}^{p}$ is an absolutely continuous module, and in particular, if $1<p<\infty$, then by Theorem $5.2, \Re\left(L_{X}^{p}\right)$ is isomorphic to $L_{X}^{p}$.

Another space whose module homomorphisms we can describe is $L_{\Gamma} \cap L_{\Gamma}^{p}$, $p \in(1, \infty]$, which we look at through the eyes of $K=C_{\Gamma}+L_{\Gamma}^{q}$, where $1 / p+1 / q=1$. Now $K$ is the linear span of $\left\{h+k: h \in C_{\Gamma}, k \in L_{\Gamma}^{q}\right\}$. Under the norm $\|j\|=\inf \left\{\|h\|+\|k\|: h \in C_{\Gamma}, k \in L_{\Gamma}^{q}, j=h+k\right\}$ and under the convolution defined by

$$
f * j(\sigma)=\int_{\Gamma} f(\tau) j\left(\tau^{-1} \sigma\right) d \tau, \quad \text { 1.a.e. } \sigma \in \Gamma,
$$

$K$ becomes an absolutely continuous $L_{\Gamma}$ module. The dual space $K^{*}=L_{\Gamma} \cap L_{\Gamma}^{p}$ has for its norm $\|g\|=\max \left(\|g\|_{1},\|g\|_{p}\right)$ (see [7, Theorem 5]). The convolution in $K^{*}$, defined by the familiar formula in (3.1) can be reduced to the formula

$$
f * g(\sigma)=\int_{\Gamma} f(\tau) g\left(\tau^{-1} \sigma\right) d \sigma \quad\left(f \in L_{\Gamma}, g \in L_{\Gamma} \cap L_{\Gamma}^{p}, \sigma \in \Gamma\right) .
$$

By Theorem 5.2, $\Re\left(L_{\Gamma} \cap L_{\Gamma}^{p}\right)$ is canonically isomorphic to $L_{\Gamma} \cap L_{\Gamma}^{p}$.

There is a connection between $K_{\text {abs }}$ and $\Re(K)$ deeper than a superficial appraisal might reveal. It becomes apparent if we consider $K \rightarrow K_{\text {abs }}$ and $\Re$ as functors in the category of all $L_{\Gamma}$ modules with continuous module homomorphisms as morphisms. It is obvious that $\Re$ is related to the well-known functor Hom in the category of all modules over a ring. Writing $L$ instead of $L_{\Gamma}$, in homological language we may denote $\Re(K)$ by $\operatorname{Hom}_{L}(L, K)$. Less obvious is the analogy between the functor $K \rightarrow K_{\mathrm{abs}}$ and the tensor product, reflected in the following theorem.

5.3. TheOREM. Let $K$ be an $L_{\Gamma}$ module. Then for any $L_{\Gamma}$ module $K^{\prime}$ and any continuous bilinear map $T: L_{\Gamma} \times K \rightarrow K^{\prime}$ that has the property $T(f * g, k)$ $=f * T(g, k)=T(g, f * k)$, there is a unique continuous homomorphism $T^{\prime}$ from $K_{\mathrm{abs}}$ into $K^{\prime}$ such that the diagram

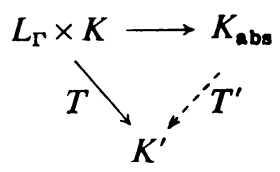


is commutative (where the horizontal arrow represents the module composition $(f, k) \rightarrow f * k)$.

Proof. Let $\left(u_{i}\right)_{i \in I}$ be an approximate identity in $L_{\Gamma}$. Take $k \in K_{\mathrm{abs}}$. There exist $f \in L_{\Gamma}$ and $j \in K$ such that $f * j=k$. Then

$$
\begin{aligned}
T(f, j) & =\lim _{i} T\left(f * u_{i}, j\right) \\
& =\lim _{i} T\left(u_{i}, f * j\right)=\lim _{i} T\left(u_{i}, k\right) .
\end{aligned}
$$

Thus we can define a linear $T^{\prime}: K_{\mathrm{abs}} \rightarrow K^{\prime}$ by $T^{\prime}(k)=\lim _{i} T\left(u_{i}, k\right)$, for $k \in K_{\text {abs }}$. The rest is straightforward.

Thus it looks reasonable to write $K_{\mathrm{abs}}=L \otimes_{L} K$. In this terminology, Theorem 5.2 takes the form

$\operatorname{Hom}_{L}\left(L, \operatorname{Hom}_{C}(K, C)\right)=\operatorname{Hom}_{C}\left(L \otimes_{L} K, C\right), \quad C$ the complex numbers,

which is a well-known formula in the algebraic theory. A theory relating Banach module homomorphisms to tensor product theory has been begun by Máté [8] and developed systematically by Rieffel [9].

6. Module homomorphisms from $L_{\Omega}$ to $L_{Y}$. As we saw in $\S 5$ there is a natural embedding $\Re\left(L_{X}\right) \rightarrow M(X)$. In the sequel we identify each $R \in \Re\left(L_{X}\right)$ with the corresponding element of $M(X)$; thus $\Re\left(L_{X}\right)=N \subseteq M(X)$. Let $\Omega \subseteq \Gamma$ and $Y \subseteq X$ be measurable. We are going to consider those $\mathfrak{R}\left(L_{X}\right)$-module homomorphisms $L_{\Gamma} \rightarrow L_{X}$ which map $L_{\Omega}$ into $L_{Y}=\left\{f \in L_{X}: f=0\right.$ a.e. outside $\left.Y\right\}$. We denote the collection of such homomorphisms by $\Re_{\Omega, Y}$. To aid the discussion we make the following definition.

6.1. Definition. Let $A_{\Omega, Y}=\{x \in X: \sigma x \in Y$ for locally almost all $\sigma \in \Omega\}$. We note that $A_{\Omega, Y}$ is measurable, by Theorem 3.15 of [5].

6.2. LeMMA. If $\mu \in \mathfrak{R}\left(L_{X}\right)$ and if $\operatorname{supp} \mu \subseteq A_{\Omega, Y}$, then $\mu \in \Re_{\Omega, Y}$.

Proof. We need to show that $L_{\Omega} * \mu \subseteq L_{Y}$. To do that, we let $k \in L_{X}^{\infty}$ be such that $\left.k\right|_{Y}=0$, and we let $f \in L_{\Omega}$. We will show that $f * \mu(k)=0$. For any $x \in \operatorname{supp} \mu \subseteq A_{\Omega, Y}$, we have $\sigma x \in Y$ for locally almost all $\sigma \in \Omega$, so that $k(\sigma x)=0$ l.a.e. on $\Omega$, resulting in $\int_{\Gamma} f(\sigma) k(\sigma x) d \sigma=0$. Therefore

$$
\begin{aligned}
(f * \mu)(k) & =\int_{X} \int_{\Gamma} f(\sigma) k(\sigma x) d \sigma d \mu(x) \\
& =\int_{\text {supp } \mu} \int_{\Gamma} f(\sigma) k(\sigma x) d \sigma d \mu(x)=0,
\end{aligned}
$$

which completes the proof.

In the event that $Y$ is closed in $X$, we can give a complete description of $\Re_{\Omega, Y}$.

6.3. THEOREM. If $Y$ is closed in $X$, then $\Re_{\Omega, Y}=\left\{\mu \in M(X): \mu \in \Re\left(L_{X}\right)\right.$ and $\left.\operatorname{supp} \mu \subseteq A_{\Omega, Y}\right\}$. 
Proof. Because of Lemma 6.2 all we must prove is that if $\mu \in \Re_{\Omega, Y}$, then supp $\mu \subseteq A_{\Omega, Y}$. By assumption, $L_{\Omega} * \mu \subseteq L_{Y}$. Then for any $k \in C_{X}$ such that $\left.k\right|_{Y}=0$, we have $(f * \mu)(k)=0$ for all $f \in L_{\Omega}$. Let $\sigma \in d \Omega$, and let $\left(u_{i}\right)_{i \in I}$ be an approximate

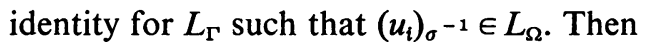

$$
\begin{aligned}
0 & =\left(\left(u_{i}\right)_{\sigma^{-1}} * \mu, k\right)=\left(\mu,\left(\left(u_{i}\right)_{\sigma}^{-1}\right)^{\prime} * k\right) \\
& =\left(\mu,\left(u_{i}^{\prime}\right)^{\sigma} * k\right)=\left(\mu,\left(u_{i}\right)^{\prime} * k_{\sigma}\right)
\end{aligned}
$$

which converges to $\mu\left(k_{\sigma}\right)$ because $C_{X}$ is absolutely continuous. Since $\sigma^{-1} Y$ is closed, supp $\mu \subseteq \sigma^{-1} Y$, for any $\sigma \in d \Omega$. But $\bigcap_{\sigma \in d \Omega} \sigma^{-1} Y \subseteq A_{\Omega, Y}$. Hence supp $\mu \subseteq A_{\Omega, Y}$.

6.4. Corollary. If $Y$ is closed in $X$, then $A_{\Omega, Y}=\bigcap_{\sigma \in d \Omega} \sigma^{-1} Y$, and hence $A_{\Omega, Y}$ is closed.

Recall that we have identified $\Re\left(L_{X}\right)$ with a subspace $N=\left\{\mu \in M(X): L_{\Gamma} * \mu \subseteq L_{X}\right\}$ of $M(X)$. Obviously this $N$ should play an important role in our discussion of $A_{\Omega, Y}$. Under the restriction that $N=M(X)$, it is not hard to prove that $A_{\Omega, Y}=A_{\Omega, Y^{\prime}}$ if $Y=Y^{\prime}$ l.a.e. (see the implication (i) $\Rightarrow$ (iii) of Theorem 5.6 in [4]). Without the restriction this is not true, as the following example shows: $\Gamma=\{1\}, Y=Y^{\prime}$ only 1.a.e. (However, from $Y=Y^{\prime}$ l.a.e. it always follows that $A_{\Omega, Y}=A_{\Omega, Y^{\prime}}$ l.a.e.) Under the condition that $N=M(X)$ we have a neater conclusion for Theorem 6.3.

\subsection{Corollary. If $Y$ is closed in $X$ and if $N=M(X)$, then}

$$
\Re_{\Omega, Y}=\left\{\mu \in M(X): \operatorname{supp} \mu \subseteq A_{\Omega, Y}\right\} .
$$

Let $\Omega \subseteq \Gamma$ be measurable and such that $L_{\Omega}$ is a subalgebra of $L_{\Gamma}$. For any Banach module $K$ over $L_{\Omega}$ we denote by $\Re_{\Omega}(K)$ the space of all continuous module homomorphisms $L_{\Omega} \rightarrow K$ (since every $L_{\Gamma}$ module is an $L_{\Omega}$ module this notation is consistent with our earlier use of the symbol $\Re_{\Omega}(K)$ ).

In particular we consider measurable subsets $Y$ of $X$ for which $L_{\Omega} * L_{Y} \subseteq L_{Y}$. For such $Y, L_{Y}$ is an $L_{\Omega}$ module. Theorem 4.3 gives an injection $\Re_{\Omega}\left(L_{Y}\right)$ $\rightarrow \prod_{J \in \mathscr{J}(d \Omega)} \Re_{\Omega \cap J, Y}$. In case $\mathscr{J}(d \Omega)$ consists of only one element, $\Re_{\Omega}\left(L_{Y}\right)$ may be identified with $\Re_{\Omega, Y}$. Then by Lemma $6.2, \Re_{\Omega}\left(L_{Y}\right) \supseteq\left\{\mu \in M(X): \operatorname{supp} \mu \subseteq A_{\Omega, Y}\right\}$ and if $N=M(X)$, the two sets are equal if $Y$ is closed (Corollary 6.5). If $N=M(X)$, it seems reasonable to ask whether we have equality for all $Y$, still assuming $\mathscr{J}(d \Omega)$ to contain only one element.

Now $N=M(X)$ if $X=\Gamma$, and $\mathscr{J}(d \Omega)$ contains only one element if $\Gamma$ is abelian (Corollary 4.4). T. A. Davis states a theorem affirming the inclusion $\Re_{\Omega}\left(L_{Y}\right)$ $\subseteq\left\{\mu \in M(X): \mu\right.$ is concentrated on $\left.A_{\Omega, Y}\right\}$ for the case $\Gamma$ is abelian, $X=\Gamma$, and $Y=\Omega$ (Theorem 3.5(2) in [2]). Unfortunately, however, his proof seems to be faulty.

By the same Corollary $4.4, \mathscr{J}(d \Omega)$ contains only one element if $1 \in d \Omega$. For this case F. Birtel [1] proves $\Re_{\Omega}\left(L_{Y}\right)=\left\{\mu \in M(X):\right.$ supp $\left.\mu \subseteq A_{\Omega, Y}\right\}$ under the assumptions $X=\Gamma, Y=\Omega, \Omega$ is a closed semigroup containing 1 whose interior is 
dense in $\Omega$. In Theorem 6.7 we prove $\Re_{\Omega}\left(L_{Y}\right)=\{\mu \in M(X): \mu$ is concentrated on $\left.A_{\Omega, Y}\right\}$ if $1 \in d \Omega$ and $N=M(X)$. In Corollary 6.8 we prove $\Re_{\Omega}\left(L_{Y}\right)=\{\mu \in M(X)$ : $\left.\operatorname{supp} \mu \subseteq A_{\Omega, Y}\right\}$ if $1 \in d \Omega$ and if for every $f \in L_{\Gamma}$ and $k \in L_{X}^{\infty}, \int_{\Gamma} f(\sigma) k\left(\sigma^{-1} x\right) d \sigma$ depends continuously on $x$, which is true for $X=\Gamma$.

We employ an auxiliary topology on $X$, called the orbit topology and designated by $\mathcal{O}$, which is generated by sets of the form $\Phi x, \Phi$ open in $\Gamma, x \in X$. This topology is studied in [5]. In what follows we shall use the facts that in case $N=M(X), \mathcal{O}$ coincides with the original topology of $X$ on each orbit $\Gamma x$, and that for $f \in L_{\Gamma}$ and $k \in L_{X}^{\infty}$, the function $x \rightarrow \int_{\Gamma} f(\sigma) k\left(\sigma^{-1} x\right) d \sigma$ is $\mathcal{O}$-continuous.

6.6. Lemma. In the topology $\mathcal{O}, A_{\Omega, Y}$ is closed.

Proof. Take $x \in X$. Then $x \in A_{\Omega, Y}$ if and only if $\int_{\Gamma} f(\sigma) \xi_{X \mid Y}(\sigma x) d \sigma=0$ for every $f \in L_{\Omega}$. Now

$$
\begin{aligned}
\int_{\Gamma} f(\sigma) \xi_{X \mid Y}(\sigma x) d \sigma & =\int_{\Gamma} f\left(\sigma^{-1}\right) \Delta\left(\sigma^{-1}\right) \xi_{X \backslash Y}\left(\sigma^{-1} x\right) d \sigma \\
& =\int_{\Gamma} f^{\prime}(\sigma) \xi_{X \mid Y}\left(\sigma^{-1} x\right) d \sigma
\end{aligned}
$$

is $\mathcal{O}$-continuous (see Lemma 4.9 of [5]); thus $A$ is $\mathcal{O}$-closed.

6.7. TheOREM. Let $\Omega \subseteq \Gamma, Y \subseteq X$ be measurable and such that $L_{\Omega}$ is a subalgebra of $L_{\Gamma}$, and $L_{\Omega} * L_{Y} \subseteq L_{Y}$. Assume $N=M(X)$. If $1 \in d \Omega$, then $\Re_{\Omega}\left(L_{Y}\right)=\{\mu \in M(X): \mu$ is concentrated on $\left.A_{\Omega, Y}\right\}$.

Proof. Let $\mu \in \mathfrak{R}_{\Omega}\left(L_{Y}\right)$. We note that $\Gamma x$ is a Borel set (Theorem 5.10 of [4]) for each $x \in X$. Since $\mu$ is bounded, $\mu$ is concentrated on a sigma-compact set. Inasmuch as any compact set can intersect only countably many orbits (see Lemma 4.6 of [5]), there exists a sequence $a_{1}, a_{2}, \ldots$ in $X$ such that $\mu$ is concentrated on $\bigcup_{n} \Gamma a_{n}$. For each $n$ define $\mu_{n}$ by $d \mu_{n}=\xi_{\Gamma a_{n}} d \mu$ and put $Y_{n}=Y \cap \Gamma a_{n}$. Then $L_{\Omega} * L_{Y_{n}} \subseteq L_{Y_{n}}$, $\mu=\sum \mu_{n}$ and $L_{\Omega} * \mu_{n} \subseteq L_{Y_{n}}$ for each $n$. It suffices to prove that each $\mu_{n}$ is concentrated on $A_{\Omega, Y n}$. In other words, we may assume the existence of an $a \in X$ such that $\mu$ is concentrated on $\Gamma a$ and $Y \subset \Gamma a$. Since $1 \in d \Omega$, by Theorem 5.6 of [5] $L_{\Omega} * L_{Y}=L_{Y}$. Let $\Omega_{0}=\Omega \cap d \Omega, T=\{x \in X$ : there exist compact sets $\Phi \subseteq \Omega$ and $D \subseteq Y$ such that $\left.\int_{\Gamma} \xi_{\Phi}(\sigma) \xi_{D}\left(\sigma^{-1} x\right) d \sigma>0\right\}$. Clearly $T \subseteq \Gamma a$. According to the proof of Theorem 5.5 of [5], we have $\Omega_{0}=\Omega$ 1.a.e., $T=Y$ l.a.e. and $\Omega_{0} T=T$. Then $T \subseteq A_{\Omega_{0}, T}=A_{\Omega, T}=A_{\Omega, Y}$.

Since $1 \in d \Omega, L_{\Omega}$ contains an approximate identity $\left(u_{i}\right)_{i \in I}$ of $L_{\Gamma}$. If $k \in C_{X}$ and $k=0$ on $\bar{T}$, then because $u_{i} * \mu \in L_{\Omega} * \mu \subseteq L_{Y}=L_{T}$,

$$
\mu(k)=\lim _{i} \mu\left(u_{i}^{\prime} * k\right)=\lim _{i}\left(u_{i} * \mu\right)(k)=0 .
$$

This means that supp $\mu \subseteq \bar{T}$ so that $\mu$ is concentrated on $\bar{T} \cap \Gamma a$. Now as we remarked in the preceding lemma, $A_{\Omega, Y}$ is $\mathcal{O}$-closed. Because the original topology 
and the $\mathcal{O}$-topology coincide on $\Gamma a$ this means that $A_{\Omega, Y} \cap \Gamma a$ is relatively closed in $\Gamma a$. Since $T \subseteq A_{\Omega . Y} \cap \Gamma a$ we obtain $\bar{T} \cap \Gamma a \subseteq A_{\Omega, Y} \cap \Gamma a \subseteq A_{\Omega, Y}$. Thus $\mu$ is concentrated on $A_{\Omega, Y}$.

6.8. Corollary. Let $\Omega, Y$ be as in the preceding theorem. Assume that for every $f \in L_{\Gamma}$ and every $k \in L_{X}^{\infty}, \int_{\Gamma} f(\sigma) k\left(\sigma^{-1} x\right) d \sigma$ depends continuously on $x \in X$. Now if $1 \in d \Omega$, then $\Re_{\Omega}\left(L_{Y}\right)=\left\{\mu \in M(X): \operatorname{supp} \mu \subseteq A_{\Omega, Y}\right\}$.

Proof. Then the orbit topology and the original topology are the same (Lemma 4.9 of [5]), and $A_{\Omega, Y}$ is thus closed in $X$. Furthermore, the assumption implies that $N=M(X)$ (Theorem 3.3 of [5]). Thus we can use the preceding theorem.

We have one comment: $\mu \in M(X)$ may be concentrated on $A_{\Omega, Y}$ without being supported on $A_{\Omega, Y}$. Let $\Gamma=\boldsymbol{R}$ be the additive group of the reals, $\Omega=(0, \infty)$, $X=\boldsymbol{R} \cup\{\infty\}$ the one-point compactification of $\boldsymbol{R}$, with usual action of $\Gamma$ on $X$, and $m_{X}(\{\infty\})=1, m_{X} \mid \boldsymbol{R}=$ Lebesgue measure, and $Y=(0, \infty)$. Then $A_{\Omega, Y}=[0, \infty)$. Let $\mu=\sum_{n=1}^{\infty} 2^{-n} \delta_{n}$ where $\delta_{n}$ is point mass at $n$. Then $\mu$ is concentrated on $A_{\Omega, Y}$ but not supported on $A_{\Omega, Y}$.

\section{REFERENCES}

1. F. T. Birtel, On a commutative extension of a Banach algebra, Proc. Amer. Math. Soc. 13 (1962), 815-822. MR 31 \#624.

2. T. A. Davis, The Wiener-Pitt phenomenon on semigroups, Proc. Cambridge Philos. Soc. 59 (1963), 11-24. MR 26 \#1701.

3. S. L. Gulick, T.-S. Liu and A. C. M. van Rooij, Group algebra modules. I, Canad. J. Math. 19 (1967), 133-150. MR 36 \#5712.

4. - Group algebra modules. II, Canad. J. Math. 19 (1967), 151-173. MR 36 \#5713.

5. - Group algebra modules. III, Trans. Amer. Math. Soc. 152 (1970), 561-579.

6. E. Hewitt and K. A. Ross, Abstract harmonic analysis. Vol. I: Structure of topological groups. Integration theory, group representations, Die Grundlehren der math. Wissenschaften, Band 115, Academic Press, New York and Springer-Verlag, Berlin and New York, 1963. MR 28 \#158.

7. T.-S. Liu and J.-K. Wang, Sums and intersections of Lebesgue spaces, Math. Scand. 23 (1968), 241-251.

8. L. Máté, Multipliers and topological tensor products (to appear).

9. M. A. Rieffel, Induced Banach representations of Banach algebras and locally compact groups, J. Functional Analysis 1 (1967), 443-491. MR 36 \#6544.

10. J. G. Wendel, Left centralizers and isomorphisms of group algebras, Pacific J. Math. 2 (1952), 251-261. MR 14, 246.

UNIVERSITY OF MARYLAND, College Park, Maryland 20742

UNIVERSITY OF MASSACHUSETTS, AMHERST, MASSACHUSETTS 01002

Catholic University, NiJMegen, The NetherLands 